\title{
Joint Energy and Rate Allocation for Successive Interference Cancellation in the Finite Blocklength Regime
}

\author{
Francesc Molina, Josep Sala-Álvarez, Javier Villares and Francesc Rey \\ Department of Signal Theory and Communications. Technical University of Catalonia (UPC) \\ Emails: \{francesc.molina, josep.sala, javier.villares, francesc.rey\} @upc.edu
}

\begin{abstract}
This work addresses the optimization of the network spectral efficiency (SE) under successive interference cancellation (SIC) at a given blocklength $n$. We adopt a proof-of-concept satellite scenario where network users can vary their transmission power and select their transmission rate from a set of encoders, for which decoding is characterized by a known packet error rate (PER) function. In the large-system limit, we apply variational calculus (VC) to obtain the user-energy distribution, the assigned per-user rate and the SIC decoding order maximizing the network SE under a sum-power constraint at the SIC input. We analyze two encoder sets: (i) an infinite set of encoders achieving information-theoretic finite blocklength PER results over a continuum of code rates, where the large- $n$ second order expansion of the maximal channel coding rate is used; (ii) a feasible finite set of encoders. Simulations quantify the performance gap between the two schemes.

Index Terms-Successive interference cancellation, packet error rate, finite blocklength, variational calculus, satellite
\end{abstract}

\section{INTRODUCTION}

In the machine-type communications (MTC) era, the explosive growth of data traffic has challenged non-orthogonal multiple access solutions in terms of their SE [1], [2]. In particular, satellite communication networks, for which random access protocols have been widely investigated [3], are bound to serve a vast number of low duty-cycle MTC terminals [4]. On the users' side, a combination of powerful physical layer encoders with code-division multiple access can be exploited [5], whereas at the central node, different multiuser detection (MUD) techniques with varying performance-complexity ratios can be applied [6]. S-MIM and DVB-RCS2 are two representative standards that contemplate successive decoding in their return satellite channels [7], [8]. In fact, SIC results a suitable MUD scheme due to its affordable complexity and satisfactory performance when power unbalance among users is properly exploited through power control [9], [10].

Several large-system analyses have optimized SIC performance by deriving their respective power control laws. In [11], the SINR and energy profiles at the SIC input associated with maximum SE were found to be, respectively, uniform and exponential. Capacity-achieving encoders (unbounded packet length) were employed, for which a single SIC iteration suffices to decode all users. In [12], [13], specific hard/soft SIC policies were analyzed when all users adopted the same

Work supported by project TEC2016-76409-C2-1-R (WINTER): Ministerio de Ciencia, Innovación y Universidades (Spanish administration), and the Catalan administration (AGAUR) under grants 2017 SGR 578 and FI-2018. practical encoder. This feature trades off system simplicity with network performance and load regulation may then act to guarantee a certain per-user performance point.

The present work has been focused on the finite blocklength regime, nowadays a relevant research area for state-of-the-art MTCs [14]. The maximum channel coding rate at finite packet length and fixed PER was derived in [15], and used, recently, to obtain optimum two-user conjoint transmission rates and power control policies [16]. We have considered instead, for system optimization, a satellite scenario that consists of a massive user network operating with an on-board relay transponder and a SIC ground gateway for demodulation [12]. The network users adjust their transmitted power and select an appropriate finite blocklength encoder from the available set (instead of adopting a common encoder as in [12], [13]). Our main contribution is the corresponding joint optimization of the power control law, the per-user transmission rate and the SIC decoding order that maximize the system SE when the useful received power at the satellite is set to a fixed operating point. A twofold analysis is performed. Firstly, we capitalize on results from finite blocklength information theory [15] to derive both the user-asymptotic energy and transmission rate profiles, showing what amount of performance degradation is incurred when using short packets. Secondly, we consider system optimization when users may select an encoder from a given finite set. Optimized SINR and energy profiles are shown to be piecewise continuously differentiable functions when users adopt encoders with different rates. A set of practical/optimum encoders is simulated to validate finite blocklength results, where the optimum SIC decoding order is found to correspond to decreasing user transmission rates.

This paper is organized as follows. In Section II, system model equations are presented. In Section III, power control optimization is performed. Section IV illustrates the performed simulations and concluding remarks are offered in Section V.

\section{System Setup}

We consider a multiple access scenario constituted by a large population of $1 \leq k \leq K$ low-rate network terminals, referred to as users. Data encoding and linear modulation for the $k$-th user's transmitted $n$-symbol packet, $s_{k}[0 \leq m<$ $n]$, comprises $n_{e}$ symbols from the channel encoder output plus $n_{o}$ preamble symbols. To that aim, each user chooses its own encoder from the set $\mathcal{M} \triangleq\left\{\mathcal{M}_{1}, \ldots, \mathcal{M}_{p}\right\}$ with efficiencies (or simply, rates) $\left\{R_{1}, \ldots, R_{p}\right\}$, where for each 
$\mathcal{M}_{1 \leq i \leq p}, k_{i}$ information bits get mapped to $n_{e}$ complex symbols. Spread spectrum modulation, appropriate at low peak-to-average transmission power ratios (PAPR), is adopted, using long spreading codes (their period far exceeding symbol duration) with common processing gain $N$. The aggregate signal (1) is first received by a transparent satellite:

$$
y(t)=\sum_{k=1}^{K} \sqrt{\frac{E_{\mathrm{x}}[k] h[k]}{T_{c}}} e^{j \phi[k]} \sum_{m=0}^{n-1} s_{k}[m] c_{k, m}(t-\tau[k])
$$

with $E_{\mathrm{x}}[k]$ the $k$-th user's complex transmitted symbol energy, $h[k], \phi[k]$ the $k$-th user's slowly time-varying uplink channel power gain and carrier phase offset, $c_{k, m}(t)$ the unit-norm spreading code of symbol $m$ with chip pulse shaping of rate $r_{c}=T_{c}^{-1}$ and roll-off factor $\beta$, and $\tau[k] \approx \tau$ a common userdelay under quasi-synchronous access [12], [13]. Directive antennas with line-of-sight transmission are assumed.

The satellite relay performs filtering and amplification $\left(G_{s}\right)$, frequency-shifting and re-transmission to a ground gateway station. The input-output curve of the satellite's high power amplifier, $P_{\text {out }}=f\left(P_{\text {in }}\right)$, is used to set the working point $\left(P_{\mathrm{wp}}\right)$ in the linear amplification region:

$$
P_{\mathrm{wp}}=N_{u} r_{c}(1+\beta)+\alpha\left(\frac{1}{K} \sum_{k=1}^{K} E_{\mathrm{x}}[k] h[k]\right) r_{c}
$$

with $N_{u}$ the uplink noise power spectral density (PSD), and $\alpha \triangleq K / N$ the system traffic load. At the gateway station, users are received with symbol energies $E_{s}[k] \triangleq E_{\mathrm{x}}[k] h[k] \cdot G_{s} h_{d}$, with $h_{d}$ the common downlink channel gain. Therefore, once $P_{\mathrm{wp}}$ is set, the following constraint is enforced

$$
\mathcal{C} \triangleq \alpha\left(\frac{1}{K} \sum_{k=1}^{K} E_{s}[k]\right)
$$

A critical gateway's requirement under massive access is efficient user decoding. SIC results, complexity-wise, a wellperforming MUD scheme. Specifically, an imperfect packet SIC policy [12] is considered (the non-persistent version of [17]), in which users are sequentially decoded, re-modulated (when successfully decoded) and canceled from the input signal. We reasonably assume, for system modeling, two characteristic curves known for each $\mathcal{M}_{i}$ : (i) the PER curve of the decoding system; (ii) the associated average residual energy (RE) function modeling the accuracy of estimationcancellation [13]. Respectively, $\operatorname{PER}\left[\Gamma, R_{i}\right]$ and $\varepsilon\left(\Gamma, R_{i}\right)$, both bivariate functions of the SINR $\Gamma$ and the encoder's rate $R_{i}$.

\section{A. SINR-based system model}

We develop a SINR-based system model where interference is reasonably assumed Gaussian as long as random spreading codes are employed [18]. Hence, the $k$-th user SINR is [12]

$\Gamma[k]=\frac{E_{s}[k]}{N_{t}[k]}=\frac{E_{s}[k]}{N_{0}+\frac{\theta}{N} \sum_{j<k} \epsilon_{j}(\Gamma[j], R[j]) E_{s}[j]+\frac{\theta}{N} \sum_{j>k} E_{s}[j]}(4)$

with $N_{t}[k]$ the $k$-th user's total noise plus interference term, $N_{0} \triangleq N_{u} G_{s} h_{d}$ the noise PSD at the gateway's input, $\frac{\theta}{N}$ the average pair-wise decorrelation between random spreading signatures [18], [19], and $\epsilon_{j}(\Gamma, R)$ a binary random variable linking decoding with imperfect SIC cancellation. Specifically, when the $j$-th user employs the encoder with rate $R[j] \in$ $\left\{R_{1}, \ldots, R_{p}\right\}$, it equals 1 under failed decoding (with prob. $\operatorname{PER}[\Gamma[j], R[j]])$, or the average RE value, $\varepsilon(\Gamma[j], R[j])$, under successful decoding.

\section{B. Large-system expressions}

In the user-asymptotic case, we conveniently let $K, N$ go to infinity so that the system load $\alpha$ remains fixed [11], and we define the continuous user index $t \triangleq \lim _{K \rightarrow+\infty} \frac{k}{K}$. Thus, the previous $k$-indexed variables can be substituted by the integration over an equivalent continuous profile in the user variable $t \in[0,1]$, by taking $k=t \cdot K$. Then, the asymptotic SINR profile $\Gamma(t) \triangleq E_{s}(t) / N_{t}(t)$ is [12]:

$$
\Gamma(t)=\frac{E_{s}(t)}{N_{0}+\alpha \theta \int_{0}^{t} r(\Gamma(\tau), R(\tau)) E_{s}(\tau) \mathrm{d} \tau+\alpha \theta \int_{t}^{1} E_{s}(\tau) \mathrm{d} \tau}
$$

with $E_{s}(t)$ the symbol energy profile at the gateway receiver, $r(\Gamma, R) \triangleq 1-(1-\varepsilon(\Gamma, R)) \operatorname{PSR}[\Gamma, R]$ the average value of the random variable $\epsilon_{j}(\Gamma, R)$, and $\operatorname{PSR}[\Gamma, R] \triangleq 1-\operatorname{PER}[\Gamma, R]$ denoting the Packet Success Rate function. Throughout this user-limit analysis, $\Gamma(t)$ results a deterministic profile. Hence, each user only needs to know its channel, estimated from a downlink pilot assuming reciprocity, to select both the transmitted symbol energy and encoder from an optimized allocation function. Additionally, as stated in [12], (5) can be alternatively expressed as the ordinary differential equation

$$
\nabla_{t} \log E_{s}(t)=\nabla_{t} \log \Gamma(t)-\alpha \Phi[\Gamma(t), R(t)],
$$

with $\nabla_{t}$ denoting differentiation under the user variable $t$, and $\Phi[\Gamma, R] \triangleq \theta(1-\varepsilon(\Gamma, R)) \Gamma \cdot \operatorname{PSR}[\Gamma, R]$ a known function that only depends on $\mathcal{M}_{i}$ and the adopted SIC policy. Equation (6) can be easily solved using the boundary condition $\Gamma(0)=$ $E_{s}(0) /\left(N_{0}+\theta \mathcal{C}\right)$ from the fact that the first user in the SIC decoding order is subject to full all-user interference. Thus

$$
E_{s}(t)=\Gamma(t) N_{t}(0) \exp \left(-\alpha \int_{0}^{t} \Phi[\Gamma(\tau), R(\tau)] \mathrm{d} \tau\right),
$$

with $N_{t}(0)=N_{0}+\theta \mathcal{C}$ the initial noise plus interference term.

\section{POWER CONTROL Optimization}

The scope of this Section is to perform the large-system optimization of the previous system set-up. We maximize the system SE (8) under constraint (9) and SIC demodulation (7).

$$
\begin{aligned}
\mathrm{SE} & \triangleq \frac{\alpha}{1+\beta} \int_{0}^{1} R(t) \operatorname{PSR}[\Gamma(t), R(t)] \mathrm{d} t \\
\mathcal{C} & =\alpha \int_{0}^{1} E_{s}(t) \mathrm{d} t
\end{aligned}
$$

We have analyzed two cases: (i) in Section III-A, when $\mathcal{M}$ consists of an asymptotically large set, $p \rightarrow+\infty$, of encoders with a continuous range of rates, whose performance is modeled by considering the normal approximation of the maximal channel coding rate [15]; (ii) in Section III-B, when $\mathcal{M}$ comprises a finite set of given encoders. For better readability, we have moved explicit derivations to the Appendix. 


\section{A. System optimization with $\mathcal{M}$ an infinite set of encoders}

We first consider the case in which $p$ is asymptotically large, and $\mathcal{M}$ denotes a set of best $n$-blocklength encoders in the sense of the finite blocklength information-theoretic result in [14], [15] for a tight approximation to the best PER performance of $n$-symbol encoders. We use the large$n$ second-order expansion of the maximal channel coding rate to obtain a PER curve (surface) as a bivariate function of the SINR $\Gamma$, and the system rate per unit bandwidth $R$ :

$$
\operatorname{PER}[\Gamma, R]=Q\left(\sqrt{\frac{n}{V}}\left(C-R+\frac{\log _{2}(n)}{2 n}\right)\right)
$$

$C \triangleq \log _{2}(1+\Gamma)$ stands for the AWGN channel capacity function, $V \triangleq\left(1-(1+\Gamma)^{-2}\right) \log _{2}^{2}(e)$ for the channel dispersion, and $Q(\cdot)$ the standard tail area function of the normal Gaussian probability density. We substitute (7) into (9). Hence, the following optimization problem is stated:

$$
\begin{aligned}
\max _{0 \leq t_{*} \leq 1} & \max _{R(t), \Gamma(t)} \int_{0}^{t_{*}} R(t) \operatorname{PSR}[\Gamma(t), R(t)] \mathrm{d} t \\
\text { s.t. } & \mathcal{C}=\alpha \int_{0}^{t_{*}} \Gamma(t) N_{t}(0) e^{-\alpha \int_{0}^{t} \Phi[\Gamma(\tau), R(\tau)] \mathrm{d} \tau} \mathrm{d} t
\end{aligned}
$$

A two-function VC problem over continuously differentiable $\Gamma(t)$, and the before spreading transmission rate profile $R(t)$, in $0 \leq t \leq t_{*}$ needs to be solved, jointly with scalar optimization over the user admission parameter $t_{*} \leq 1$ that performs load regulation under high system load.

The solution to our problem is such a $\Gamma_{0}(t), R_{0}(t)$ pair that simultaneously fulfils, over $0 \leq t \leq t_{*}$, the equations:

$$
\begin{aligned}
R_{0}(t) \mathrm{PSR}_{\Gamma} & =\lambda\left(N_{t}(t)-\alpha I(t) \Phi_{\Gamma}\right) \\
\mathrm{PSR}+R_{0}(t) \mathrm{PSR}_{R} & =-\lambda \alpha I(t) \Phi_{R}
\end{aligned}
$$

with $\lambda>0$ to be determined, and $\mathrm{PSR}_{\Gamma}, \Phi_{\Gamma}, \mathrm{PSR}_{R}, \Phi_{R}$ denoting partial derivatives in $\Gamma$ and $R$. Moreover, optimal load regulation starts when the last user's SINR and rate are set to $\Gamma_{0}\left(t_{*}\right)=\Gamma_{*}$ and $R_{0}\left(t_{*}\right)=R_{*}$, which solve:

$$
\operatorname{PSR}\left[\Gamma_{*}, R_{*}\right]=\Gamma_{*} \operatorname{PSR}_{\Gamma}\left[\Gamma_{*}, R_{*}\right]=-R_{*} \operatorname{PSR}_{R}\left[\Gamma_{*}, R_{*}\right]
$$

The particular case of infinite blocklength packets can be easily studied by letting $n \rightarrow+\infty$. In that case, it is straightforward to show that $\Gamma_{0}(t)=\alpha^{-1} \log \left(1+\mathcal{C} / N_{0}\right)$ in $0 \leq t \leq 1$, and that the $\mathrm{SE}$ is, as in [11]:

$$
\mathrm{SE}=\alpha \log _{2}\left(1+\alpha^{-1} \log \left(1+\mathcal{C} / N_{0}\right)\right) /(1+\beta) .
$$

\section{B. System optimization with $\mathcal{M}$ a finite set of given encoders}

Let us consider now the practical case in which $\mathcal{M}$ is finite. The known PER and RE functions associated to $\mathcal{M}_{i}$ are denoted $\operatorname{PER}\left[\Gamma, R_{i}\right]$ and $\varepsilon\left(\Gamma, R_{i}\right)$. We may consider the decoding policy in which users are demodulated in groups of users that share the same encoder. For that reason, we define the partition of users $\tau \triangleq\left\{t_{0}=0, t_{1}, \ldots, t_{p} \leq 1\right\}$ where users comprised between $t_{i}$ and $t_{i+1}$ employ $\mathcal{M}_{i}$. Note that $t_{p}$ takes the role of user admission $t_{*}$ in Section III-A. Therefore, the following triple optimization problem needs to be solved:

$$
\begin{aligned}
\max _{\mathcal{P}(\mathcal{M})} & \max _{t_{1}, \ldots, t_{p}} \max _{\Gamma(t)} \sum_{i=1}^{p} \int_{t_{i-1}}^{t_{i}} R_{i} \operatorname{PSR}\left[\Gamma(t), R_{i}\right] \mathrm{d} t \\
\text { s.t. } & \mathcal{C}=\alpha \sum_{i=1}^{p} \int_{t_{i-1}}^{t_{i}} \Gamma(t) N_{t}(0) e^{-\alpha \int_{t_{i-1}}^{t} \Phi[\Gamma(\tau), R(\tau)] \mathrm{d} \tau} \mathrm{d} t
\end{aligned}
$$

which comprises: (i) a VC problem to maximize SE under the piecewise continuously differentiable $\Gamma(t)$, (ii) univariate optimizations of user indices $0 \leq t_{1}, \ldots, t_{p} \leq 1$; and (iii) $P(\mathcal{M})$ denoting permutations of elements in $\mathcal{M}$.

The solution of the VC problem can be established by splitting (13) into the following $p$ equations in $t_{i-1}<t \leq t_{i}$ :

$$
R_{i} \operatorname{PSR}_{\Gamma}\left[\Gamma_{0}(t), R_{i}\right]=\lambda\left(N_{t}(t)-\alpha I(t) \Phi_{\Gamma}\left[\Gamma_{0}(t), R_{i}\right]\right)
$$

Optimum $t_{1}, \ldots, t_{p-1}$ are found by exhaustive search, and user admission $t_{p}=t_{*}$ is such that the last user's $\operatorname{SINR}, \Gamma\left(t_{*}\right)$, equals $\Gamma_{*}$, the solution of $\operatorname{PSR}\left[\Gamma_{*}, R_{p}\right]=\Gamma_{*} \operatorname{PSR}_{\Gamma}\left[\Gamma_{*}, R_{p}\right]$.

\section{Simulations}

We depict in Fig. 1 the network SE as a function of the system load for $\mathcal{C} / N_{0}=10 \mathrm{~dB}, \theta=1$, and $\beta=0$. In blue, the capacity bound (16). The SE in the finite blocklength regime with perfect $\operatorname{SIC}(\varepsilon(\Gamma, R)=0)$, the solution resulting from (13 - 14), is illustrated in solid lines for $n$ equal to 5000, 2000, 1000 and 498 symbols. Performance degradation is caused by non-error-free decoders, an effect mitigated as $n$ increases.

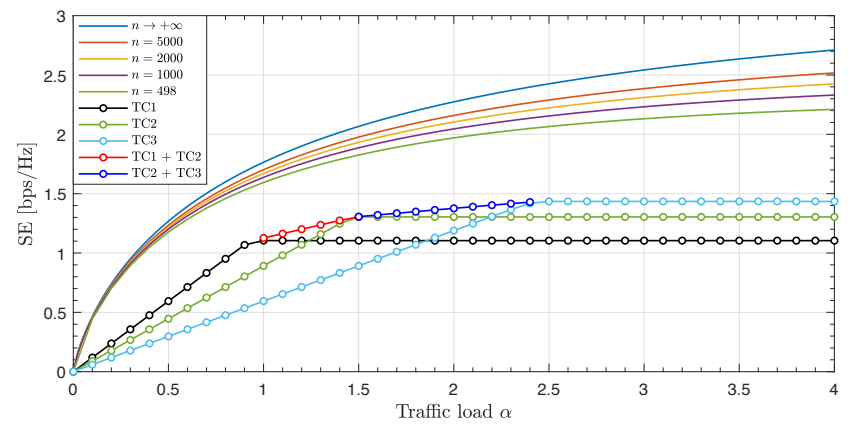

Fig. 1. SE (in bits per second and Hertz) vs. traffic load $\alpha$.

Two different analyses are performed for the case of $p=3$ encoders. Firstly, we evaluate the case when practical encoders are employed, and imperfect SIC operates. Specifically, we use the 8-state 3GPP Turbo code (TC) [20], [21] with optimized DRP interleavers [22], puncturing according to [23], QPSK modulation (for low PAPR), coding rates slightly lower (12bit tail biting) than $2 / 3$ (TC1), $1 / 2$ (TC2), and $1 / 3$ (TC3), and decoding under 10 max-log-map iterations. Packet lengths are $n=498$, with $n_{o}=48$ and $n_{e}=450$. Resulting rates are $R_{1}=1.19, R_{2}=0.89$ and $R_{3}=0.59 \mathrm{bps} / \mathrm{Hz}$.

We start by considering users sharing the same encoder, i.e. $p=1$, as in [12]. The system's SE increases until load regulation forces SE saturation (see black, green and light blue circles). Saturation loads are $\alpha_{\mathrm{TC} 1}=0.96, \alpha_{\mathrm{TC} 2}=1.50$ and $\alpha_{\mathrm{TC} 3}=2.44$. Then, when $p=3$ and $\alpha \in\left[0, \alpha_{\mathrm{TC1}}\right)$ $\left(\alpha \in\left[\alpha_{\mathrm{TC} 3},+\infty\right)\right)$, users share the $\mathcal{M}_{i}$ with highest (lowest) rate, while in the intermediate load intervals $\left[\alpha_{\mathrm{TC} 1}, \alpha_{\mathrm{TC} 2}\right)$ and $\left[\alpha_{\mathrm{TC} 2}, \alpha_{\mathrm{TC} 3}\right)$, only two encoders are employed: TC1/TC2 (red circles) and TC2/TC3 (dark blue circles), respectively. 
Secondly, in Fig. 2, we evaluate $p=3$ theoretical $n$-symbol encoders with PER curves obtained by substituting $R_{1}, R_{2}$ and $R_{3}$ in (10), namely BL1, BL2 and BL3, whilst adopting perfect SIC. The optimum SE practically attains the theoretical (green) bound. The loss incurred is due to a fraction of users sharing the same encoder, rather than employing different rates. Finally, in Fig. 3, we show the optimum symbol energy $E_{s}(t)$ and transmitted rate $R_{0}(t)$ profiles at $\alpha=2.00$.

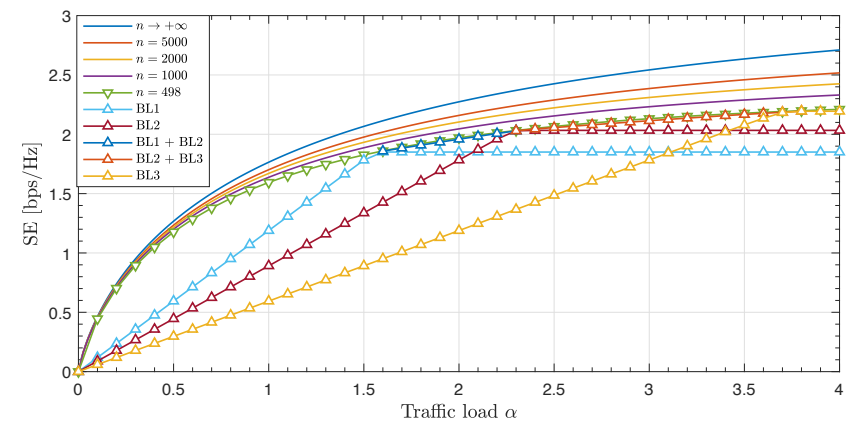

Fig. 2. SE (in bits per second and Hertz) vs. traffic load $\alpha$.

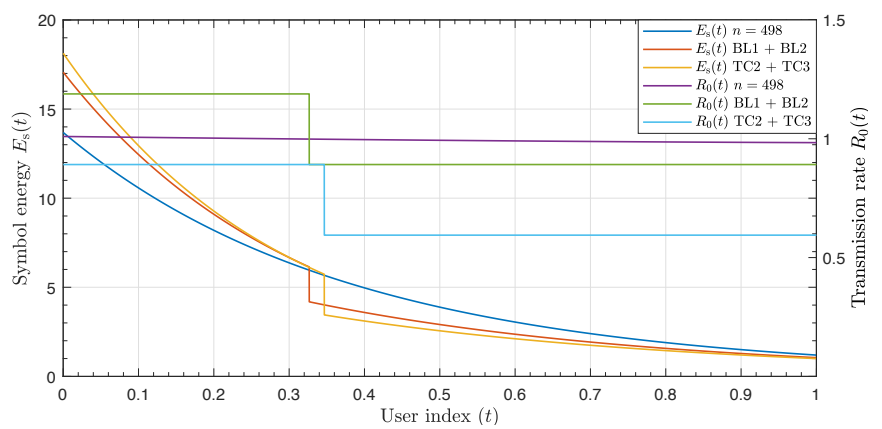

Fig. 3. $E_{s}(t)$ and $R_{0}(t)$ for the traffic load $\alpha=2.00$.

For $n=498$, the optimized $E_{s}(t)$ is practically exponential, and $R_{0}(t)$ is quasi-uniform. Users must use different encoders with similar but not identical rates. When $\mathcal{M}$ constitutes a finite set, 2-piecewise continuously differentiable profiles, $E_{s}(t)$ and $R_{0}(t)$, are found to optimize SE. Both the energy and rate are non-increasing in the SIC decoding order.

\section{CONCLUSIONS}

A multiple access satellite network where user terminals can select their encoder and vary their transmitted power to favor SIC performance has been considered. Spectral efficiency has been optimized in the large-system limit by deriving the user-energy and transmission rate profiles when the SIC gateway demodulator operates under a sum-power constraint. The optimization problem has been tackled, using Variational Calculus, in terms of the known PER function of the decoders and the specific SIC implementation. The performance in the finite blocklength regime has been studied when a large set of encoders, with rates and PER curves according to the large- $n$ second order expansion of the maximal channel coding rate, are adopted. For a set of $p=3$ practical/optimum encoders, best spectral efficiency at each load is reported when users selectively operate with at most two of the encoders, with symbol energy and transmission rate profiles that are nonincreasing piecewise continuously differentiable functions of the SIC decoding order.

\section{APPENDIX}

In this appendix, we derive the stationary point equations of the two-function VC problem $(11$ - 12) and the equations that govern the optimum $t_{*}$. First, we define the Lagrangian $\mathcal{L} \triangleq \int_{0}^{t_{*}} R(t) \operatorname{PSR}[\Gamma(t), R(t)] \mathrm{d} t-\lambda \int_{0}^{t_{*}} \Gamma(t) N_{t}(0) e^{-B(t)} \mathrm{d} t$ with $B(t)$ defined by context. Also, we consider variations:

$$
\begin{aligned}
\Gamma(t) & =\Gamma_{0}(t)+a \cdot \psi(t) \\
R(t) & =R_{0}(t)+b \cdot \phi(t)
\end{aligned}
$$

where $\Gamma_{0}(t), R_{0}(t)$ denote continuously differentiable profiles in $0 \leq t \leq t_{*}$, and $a, b$ together with $\psi(t), \phi(t)$ the magnitude and direction of the performed variations. We solve the stated VC problem in the sequel. For brevity, we write PSR and $\Phi$ instead of $\operatorname{PSR}[\Gamma(t), R(t)]$ and $\Phi[\Gamma(t), R(t)]$. Partial derivatives w.r.t. $\Gamma$ and $R$ are denoted $\operatorname{PSR}_{\Gamma}, \operatorname{PSR}_{R}$ and $\Phi_{\Gamma}, \Phi_{R}$.

\section{A. Stationary point equation of $\Gamma_{0}(t)$}

As stated in [24], for $\Gamma_{0}(t)$ to be a stationary point, it is necessary that the limit $\nabla_{a \rightarrow 0} \mathcal{L}$ vanishes for every admissible $\psi(t)$. The gradient over the first term of $\mathcal{L}$ is

$\int_{0}^{t_{*}}\left[\left(R_{0}(t) \mathrm{PSR}_{\Gamma}-\lambda N_{t}(t)\right) \psi(t)+\lambda \alpha E_{s}(t) \int_{0}^{t} \Phi_{\Gamma} \psi(\tau) \mathrm{d} \tau\right] \mathrm{d} t$.

We may apply integration by parts with $u=\int_{0}^{t} \Phi_{\Gamma} \psi(\tau) \mathrm{d} \tau$ and $\mathrm{d} v=E_{s}(t) \mathrm{d} t=-\mathrm{d} I$, where $I(t) \triangleq \int_{t}^{t_{*}} E_{s}(\tau) \mathrm{d} \tau$. Hence, the following must be set to zero

$$
\int_{0}^{t_{*}}\left[R_{0}(t) \mathrm{PSR}_{\Gamma}-\lambda\left(N_{t}(t)-\alpha \int_{t}^{t_{*}} E_{s}(\tau) \mathrm{d} \tau \Phi_{\Gamma}\right)\right] \psi(t) \mathrm{d} t .
$$

Applying the Fundamental Lemma of the Calculus of Variations (FLCV) [24], we get

$$
R_{0}(t) \mathrm{PSR}_{\Gamma}=\lambda\left(N_{t}(t)-\alpha I(t) \Phi_{\Gamma}\right) \quad 0 \leq t \leq t_{*}
$$

\section{B. Stationary point equation of $R_{0}(t)$}

The limit $\nabla_{b \rightarrow 0} \mathcal{L}$ must vanish for every admissible $\phi(t)$. Over $\mathcal{L}$, we apply the product rule at $R(t)$ and PSR. We get $\int_{0}^{t_{*}}\left[\mathrm{PSR}+R_{0}(t) \mathrm{PSR}_{R}\right] \phi(t) \mathrm{d} t+\lambda \alpha \int_{0}^{t_{*}} E_{s}(t) \int_{0}^{t} \Phi_{R} \phi(\tau) \mathrm{d} \tau \mathrm{d} t$ We follow the same procedure described in Section A. Integrating by parts, and using the FLCV [24], we obtain

$$
\mathrm{PSR}+R_{0}(t) \mathrm{PSR}_{R}=-\lambda \alpha I(t) \Phi_{R} \quad 0 \leq t \leq t_{*}
$$

\section{Optimum $t_{*}$}

The optimum user index, $t_{*}$, is obtained by setting $\nabla_{t_{*}} \mathcal{L}=$ 0 . This gives $R_{0}\left(t_{*}\right) \operatorname{PSR}\left[\Gamma_{0}\left(t_{*}\right), R_{0}\left(t_{*}\right)\right]=\lambda E_{s}\left(t_{*}\right)$, that we combine with the stationary point equations (22) and (23) at $t_{*}$ by setting $I\left(t_{*}\right)=0$. We obtain

$$
\left.\mathrm{PSR}\right|_{t_{*}}=\left.\Gamma_{0}\left(t_{*}\right) \mathrm{PSR}_{\Gamma}\right|_{t_{*}}=-\left.R_{0}\left(t_{*}\right) \mathrm{PSR}_{R}\right|_{t_{*}}
$$

where we write $\left.\right|_{t_{*}}$ to indicate evaluation at $\Gamma_{0}\left(t_{*}\right)$ and $R_{0}\left(t_{*}\right)$. 


\section{REFERENCES}

[1] L. Dai, B. Wang, Y. Yuan, S. Han, I. Chih-Lin, and Z. Wang, "Nonorthogonal multiple access for 5G: solutions, challenges, opportunities, and future research trends," IEEE Commun. Mag., vol. 53, no. 9, pp. 74-81, September 2015

[2] Z. Ding, X. Lei, G. K. Karagiannidis, R. Schober, J. Yuan, and V. K. Bhargava, "A survey on non-orthogonal multiple access for $5 \mathrm{G}$ networks: Research challenges and future trends," IEEE J. Sel. Areas Commun., vol. 35, no. 10, pp. 2181-2195, October 2017.

[3] R. De Gaudenzi, O. Del Río Herrero, G. Gallinaro, S. Cioni, and P. Arapoglou, "Random access schemes for satellite networks, from VSAT to M2M: a survey," Int. J. Satellite Communications Networking, vol. 36, pp. 66-107, 2018.

[4] A. Mengali, R. De Gaudenzi, and Č. Stefanović, "On the modeling and performance assessment of random access with SIC," IEEE J. Sel. Areas Commun., vol. PP, no. 99, pp. 1-1, February 2018.

[5] R. De Gaudenzi and O. del Río Herrero, "Advances in random access protocols for satellite networks," in 2009 Int. Workshop on Satellite and Space Communications, September 2009, pp. 331-336.

[6] S. Verdú, Multiuser detection. Cambridge University Press, 1998.

[7] ETSI EN, "Digital video broadcasting (DVB); Second generation DVB Interactive satellite system (DVB-RCS2); Part 2: Lower layers for satellite standard," ETSI EN 301 545-2 V1.2.1, April 2014.

[8] TS ETSI, "Satellite Earth Stations and Systems; Air interface for S-band mobile interactive multimedia (S-MIM)."

[9] A. Agrawal, J. G. Andrews, J. M. Cioffi, and T. Meng, "Iterative power control for imperfect successive interference cancellation," IEEE Trans. Wireless Commun., vol. 4, no. 3, pp. 878-884, May 2005.

[10] N. Benvenuto, G. Carnevale, and S. Tomasin, "Joint power control and receiver optimization of CDMA transceivers using successive interference cancellation," IEEE Trans. Commun., vol. 55, no. 3, pp. 563-573, March 2007.

[11] D. V. Djonin and V. K. Bhargava, "Asymptotic Analysis of the Conventional Decision Feedback Receiver in Fading Channels," IEEE Trans. Wireless Commun., vol. 2, no. 5, pp. 1066-1078, September 2003.

[12] J. Sala-Álvarez, F. Rey, J. Villares, and F. Molina, "Minimum PER user-energy profile for massive SIC receivers under an average energy constraint," in 2017 IEEE 18th Int. Workshop on Signal Processing Advances in Wireless Communications (SPAWC), July 2017, pp. 1-6.
[13] F. Molina, J. Sala-Álvarez, J. Villares, and F. Rey, "Optimal power control law for equal-rate DS-CDMA networks governed by a successive soft interference cancellation scheme," in 2018 IEEE International Conference on Acoustics, Speech and Signal Processing (ICASSP), 2018 April, pp. 1-5.

[14] G. Durisi, T. Koch, and P. Popovski, "Toward massive, ultra-reliable, and low-latency wireless communication with short packets," Proceedings of the IEEE, vol. 104, no. 9, pp. 1711-1726, September 2016.

[15] Y. Polyanskiy, H. V. Poor, and S. Verdú, "Channel coding rate in the finite blocklength regime," IEEE Trans. Inf. Theory, vol. 56, no. 5, pp. 2307-2359, May 2010.

[16] X. Sun, S. Yan, N. Yang, Z. Ding, C. Shen, and Z. Zhong, "Shortpacket downlink transmission with non-orthogonal multiple access," IEEE Trans. Wireless Commun., pp. 1-15, April 2018.

[17] R. De Gaudenzi, O. del Río Herrero, and G. Gallinaro, "Enhanced spread ALOHA physical layer design and performance," Int. Journal on Satellite Communications and Networking, vol. 32, no. 6, pp. 457473, 2014.

[18] S. Verdú and S. Shamai (Shitz), "Spectral efficiency of CDMA with random spreading," IEEE Trans. Inf. Theory, vol. 45, no. 2, pp. 622640, March 1999.

[19] R. M. Buehrer, "Equal BER performance in linear successive interference cancellation for CDMA systems," IEEE Trans. Commun., vol. 49, no. 7, pp. 1250-1258, July 2001.

[20] TS ETSI, "Universal mobile telecommunications systems (UMTS); Multiplexing and channel coding (FDD)," ETSI TS 125212 v12.1.0 Release 12, January 2015.

[21] ETSI TS, "LTE; Evolved Universal Terrestrial Radio Access (E-UTRA); Multiplexing and channel coding," ETSI TS 136212 V12.2.0 Release 12, October 2014

[22] S. Crozier, R. Kerr, and J. Lodge, "Adaptive coding and modulation for return satellite links using binary turbo coding," in 2010 5th Advanced Satellite Multimedia Systems Conference and the 11th Signal Processing for Space Communications Workshop, September 2010, pp. 98-105.

[23] S. Crozier and P. Guinand, "High-performance low-memory interleaver banks for turbo-codes," February 2005, US Patent 6,857,087.

[24] I. M. Gelfand, R. A. Silverman et al., Calculus of variations. Courier Corporation, 2000. 